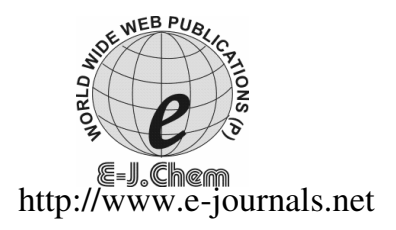

ISSN: 0973-4945; CODEN ECJHAO

E-Journal of Chemistry

$2011,8(2), 535-540$

\title{
Fast and Efficient Method for Imination of $N$-Aminorhodanine Using Inorganic Solid Support under Microwave Irradiation and Classical Heating
}

\author{
MASOUMEH TABATABAEE* ${ }^{*}$ MAJID M. HERAVI, \\ MAHBOUBEH SHARIF $^{\#}$ and FATEMEH ESFANDIYARI ${ }^{\S}$ \\ ${ }^{*}$ Scientific Society of Nanotechnology \\ Islamic Azad University, Yazd Branch, Yazd, Iran \\ Department of Chemistry \\ School of Sciences, Alzahra University, Tehran, Iran \\ "Department of Chemistry \\ Islamic Azad University- Qom Branch, Qom, Iran \\ ${ }^{\S}$ Department of Chemistry \\ Islamic Azad University- Yazd Branch, Yazd, Iran \\ tabatabaee45m@yahoo.com
}

Received 16 June 2010; Revised 13 September 2010; Accepted 6 November 2010

\begin{abstract}
Reaction of $\mathrm{N}$-aminorhodanine with various aldehydes under classical heating (in various solvents, using hydrochloric acid or acetic acid as catalyst) or by using $\mathrm{Al}_{2} \mathrm{O}_{3}$ under microwave irradiation in solventless system led to the corresponding imines ( $\mathbf{2}$ or $\mathbf{3}$ ). All the compounds have been characterized by elemental analyses, NMR and IR spectroscopy. Results indicate that treatment of $\mathrm{N}$-aminorhodanine with aldehydes in acetonitrile as solvent or under solventless system led to the corresponding imines (2a-2e), while reactions were performed in solvent such as methanol 3a-3e was formed as products of ring cleavage and imination.
\end{abstract}

Keywords: Imines, Microwave irradiation, Inorganic supports.

\section{Introduction}

There is considerable interest in the chemistry of iminic compounds ${ }^{1-4}$. Compounds containing imine bond with different donor atoms, also use in analytical applications and metal coordination ${ }^{5-7}$. Since many compounds containing sulfur and nitrogen atoms are antihypertensive, analgesic, antiinflammatory, sedative or fungicidal, synthesis of the corresponding derivatives could be of interest from the viewpoint of chemical reactivity and biological activity ${ }^{8}$. 
The use of microwave irradiation in organic synthesis has become increasingly popular within the pharmaceutical and academic arenas, because it is a new technology to accelerate the organic reaction dramatically. Microwave irradiation enhanced chemical reactions especially under solvent-free conditions have attracted much attention ${ }^{9}$. In continuation of our recent work on microwave-assisted reactions under solvent-free conditions for the synthesis of some iminic compounds derived from 4-amino-6-methyl-1,2,4-triazine-3thione-5 (2H)-one (AMTTO) and 4-amino-5-methyl-1, 2,4-triazole-3 (4H)-thione (AMTT) $)^{8,10}$. In this communication, we wish to report our results for the synthesis of various iminic compouds derived from $\mathrm{N}$-aminorhodanine under classical heating and using $\mathrm{Al}_{2} \mathrm{O}_{3}$ as inorganic support, under microwave irradiation in solventless system.

\section{Experimental}

FT-IR spectra bruker tensor 27 spectrometer ( $\mathrm{KBr}$ pellets, nujol mulls, 4000-400 $\mathrm{cm}^{-1}$ ) was used. ${ }^{1} \mathrm{H}$ and ${ }^{13} \mathrm{CNMR}$ were recorded on a Brucker (AX-200) $300 \mathrm{MH}$ spectrometer using TMS as an external standard. Elemental analyses were performed using a Costech ECS 4010 CHNS-O analyzer. All chemicals and solvents were purchased from Merck and Fluka and were used without further purification or drying. $\mathrm{Al}_{2} \mathrm{O}_{3}$ active acidic (Merck) was used as solid support.

General procedure for the reaction of $\mathrm{N}$-aminorhodanine with various aldehydes under classical heating

A solution of $\mathrm{N}$-aminorhodanine $(2 \mathrm{mmol})$ in $\left(\mathrm{CH}_{3} \mathrm{CN}\right.$ or $\left.\mathrm{CH}_{3} \mathrm{OH}, 10 \mathrm{~mL}\right)$ was treated with aldehydes in a molar ratio of 1:1.5 and the resulting mixture was acidified by $37 \%$ hydrochloric acid ( 3 drops) or acetic acid ( 8 drops). The reaction mixture was refluxed for indicated time (Table 1). The solid residue was filtered, washed with cold solvent $(10 \mathrm{~mL})$ to afford the compound 2 (in $\mathrm{CH}_{3} \mathrm{CN}$ and $\mathbf{3}$ (in $\mathrm{CH}_{3} \mathrm{OH}$ ).

General procedures for the reaction of $\mathrm{N}$-aminorhodanine with various aldehydes using inorganic solid supports under microwave irradiation

$N$-aminorhodanine $(1 \mathrm{mmol})$ and aldehyde were taken in a molar ratio of 1:1.5 was mixed with $\mathrm{Al}_{2} \mathrm{O}_{3}(1 \mathrm{~g})$ in a beaker. The beaker was placed in microwave oven for appropriate time. The reaction mixture was dissolved in acetone/THF $(1: 1$ ratio) the solid residue was filtered. The clear filtrate was kept to give the corresponding imine (2a-2e).

\section{Selected physical data}

\section{3-(4-Chlorobenzylideneamino)-2-thioxothiazolodin-4-one (2a)}

This compound was obtained as pale yellow crystalline, mp 139-141 ${ }^{\circ} \mathrm{C}$; IR $\widetilde{v}$ (KBr disc): CO 1700, CN (imine) 1601(s) $\mathrm{cm}^{-1} ;{ }^{1} \mathrm{H}$ NMR (d $\left.6^{-} \mathrm{DMSO}\right): \delta=4.37\left(\mathrm{~s}, 2 \mathrm{H}, \mathrm{CH}_{2}\right), 7.64$ $(\mathrm{d}, 2 \mathrm{H}, \mathrm{Ar}, \mathrm{J}=6.82), 7.94(\mathrm{~d}, 2 \mathrm{H}, \mathrm{Ar}, \mathrm{J}=8.81), 8.78(\mathrm{~s}, 1 \mathrm{H}, \mathrm{CH}=\mathrm{N}) \mathrm{ppm} .{ }^{13} \mathrm{C} \mathrm{NMR}$ $\left(\mathrm{d}_{6}-\mathrm{DMSO}\right): \delta=35.65$ (1C, $\mathrm{CH}_{2}$ (amine fragment), 130.30, 131.39, 131.57, 138.67(6C, $\mathrm{Ar}), 169.94(1 \mathrm{C}, \mathrm{C}=\mathrm{N}$, imine), $170.44(\mathrm{C}=\mathrm{O}), 197.78(\mathrm{C}=\mathrm{S}) \mathrm{ppm}$. Anal. Calcd. for $\mathrm{C}_{10} \mathrm{H}_{7} \mathrm{ClN}_{2} \mathrm{OS}_{2}(\mathrm{M}=270.5)$ : C, 44.36, H, 2.58, N, 10.38. Found: C, 44.18, H, 2.41, N, 10.26. 3-(3-Nitrobenzylideneamino)-2-thioxothiazolodin-4-one (2b)

Mp 283-286 ${ }^{\circ} \mathrm{C}$; IR $\widetilde{v}$ (KBr disc): $\mathrm{CO} 1729, \mathrm{CN}\left(\right.$ imine) $1610(\mathrm{~s}), \mathrm{NO}_{2}(1523,1345) \mathrm{cm}^{-1} ;{ }^{1} \mathrm{H}$ NMR (d $6^{-}$DMSO): $\delta=4.40\left(\mathrm{~s}, 2 \mathrm{H}, \mathrm{CH}_{2}\right), 7.85(\mathrm{t}, 1 \mathrm{H}, \mathrm{Ar}), 8.35(\mathrm{~d}, 1 \mathrm{H}, \mathrm{Ar}, \mathrm{j}=4.2), 8.45$ $(\mathrm{d}, 1 \mathrm{H}, \mathrm{Ar}, \mathrm{J}=4.8), 8.72(\mathrm{~s}, 1 \mathrm{H}, \mathrm{Ar}), 9.01(\mathrm{~s}, 1 \mathrm{H}, \mathrm{CH}=\mathrm{N}) \mathrm{ppm},{ }^{13} \mathrm{C} \mathrm{NMR}\left(\mathrm{d}_{6}-\mathrm{DMSO}\right)$ : $\delta=35.65\left(\mathrm{C}, \mathrm{CH}_{2}\right.$ (amine fragment), 123.86, 128.04, 131.83, 134.32, 135.57, 149.15 (6C, Ar), $168.39(\mathrm{C}, \mathrm{C}=\mathrm{N}$, imine $), 170.36(\mathrm{C}=\mathrm{O}), 197.88(\mathrm{C}=\mathrm{S})$ ppm. Anal. Calcd. for $\mathrm{C}_{10} \mathrm{H}_{7} \mathrm{~N}_{3} \mathrm{O}_{3} \mathrm{~S}_{2}(\mathrm{M}=281)$ : C, 42.7, H, 2.49, N, 14.94. Found: C, 42.58, H, 2.29, N, 14.63. 


\section{3-(4-Methoxybenzylideneamino)-2-thioxothiazolodin-4-one (2c)}

Mp 167-169 ${ }^{\circ} \mathrm{C}$; IR $\widetilde{v}$ (KBr disc): CO 1705, CN(imine) $1616(\mathrm{~s}) \mathrm{cm}^{-1} ;{ }^{1} \mathrm{H}$ NMR (d $6^{-}$DMSO): $\delta=3.86\left(\mathrm{~s}, 3 \mathrm{H}, \mathrm{OCH}_{3}\right), 4.35\left(\mathrm{~s}, 2 \mathrm{H}, \mathrm{CH}_{2}\right), 7.11(\mathrm{~d}, 1 \mathrm{H}, \mathrm{Ar}, \mathrm{J}=5.4), 7.86(\mathrm{~d}, 1 \mathrm{H}, \mathrm{Ar}, \mathrm{J}=5.1)$, $8.60(\mathrm{~s}, 1 \mathrm{H}, \mathrm{CH}=\mathrm{N}) \mathrm{ppm},{ }^{13} \mathrm{C} \mathrm{NMR}\left(\mathrm{d}_{6}-\mathrm{DMSO}\right): \delta=35.55\left(\mathrm{C}, \mathrm{CH}_{2}\right.$ (amine fragment), $56.45\left(\mathrm{C}, \mathrm{OCH}_{3}\right), 115.56,125.17,131.82,164.03(6 \mathrm{C}, \mathrm{Ar}), 170.63(\mathrm{C}, \mathrm{C}=\mathrm{N}$, imine $), 171.15$ $(\mathrm{C}=\mathrm{O}), 197.69(\mathrm{C}=\mathrm{S}) \mathrm{ppm}$. Anal. Calcd. for $\mathrm{C}_{11} \mathrm{H}_{10} \mathrm{~N}_{2} \mathrm{O}_{2} \mathrm{~S}_{2}(\mathrm{M}=266)$ : C, 49.62, $\mathrm{H}, 3.75, \mathrm{~N}$, 10.53. Found: C, 49.35, H, 3.37, N, 10.33 .

\section{3-(2-Hydroxy-3-methoxybenzylideneamino)-2-thioxothiazolodin-4-one (2d)}

Mp 210-213 ${ }^{\circ} \mathrm{C}$; IR $\widetilde{v}$ (KBr disc): CO 1669, CN(imine) $1598(\mathrm{~s}) \mathrm{cm}^{-1} ;{ }^{1} \mathrm{H}$ NMR (d $\mathrm{d}^{-}$DMSO): $\delta=3.82\left(\mathrm{~s}, 3 \mathrm{H}, \mathrm{OCH}_{3}\right), 4.31\left(\mathrm{~s}, 2 \mathrm{H}, \mathrm{CH}_{2}\right), 6.91(\mathrm{~m}, 1 \mathrm{H}, \mathrm{Ar}), 7.18(\mathrm{~d}, 1 \mathrm{H}, \mathrm{Ar}, \mathrm{J}=7.5), 7.39$ $(\mathrm{d}, 1 \mathrm{H}$, Ar, $\mathrm{J}=7.96), 9.15(1 \mathrm{H}, \mathrm{CH}=\mathrm{N}), 10.16(1 \mathrm{H}, \mathrm{OH}) \mathrm{ppm}$. Anal. Calcd. for $\mathrm{C}_{11} \mathrm{H}_{10} \mathrm{~N}_{2} \mathrm{O}_{3} \mathrm{~S}_{2}(\mathrm{M}=282)$ : C, 46.81, H, 3.55, N, 9.93. Found: C, 46.76, H, 3.48, N, 9.86.

3-(4-Hydroxybenzylideneamino)-2-thioxothiazolodin-4-one (2e)

Mp 264-265 ${ }^{\circ} \mathrm{C}$; IR $\widetilde{v}$ (KBr disc): $\mathrm{OH} 3413, \mathrm{CO} 1697, \mathrm{CN}$ (imine) 1574(s) $\mathrm{cm}^{-1} ;{ }^{1} \mathrm{H}$ NMR $\left(\mathrm{d}_{6^{-}} \mathrm{DMSO}\right): \delta=5.93\left(\mathrm{~s}, 2 \mathrm{H}, \mathrm{CH}_{2}\right), 6.93(\mathrm{~d}, 2 \mathrm{H}, \mathrm{Ar}, \mathrm{J}=6.42), 7.53(\mathrm{~d}, 2 \mathrm{H}, \mathrm{Ar}, \mathrm{J}=6.63)$, $7.67(\mathrm{~s}, 1 \mathrm{H}, \mathrm{CH}=\mathrm{N}), 10.50(1 \mathrm{H}, \mathrm{OH}) \mathrm{ppm},{ }^{13} \mathrm{C} \mathrm{NMR}\left(\mathrm{d}_{6}-\mathrm{DMSO}\right): \delta=39.8\left(\mathrm{C}, \mathrm{CH}_{2}\right.$ (amine fragment), 116.60, 123.10, 131.75, $133.95(6 \mathrm{C}, \mathrm{Ar}), 134.84(\mathrm{C}, \mathrm{C}=\mathrm{N}$, imine $), 171.51(\mathrm{C}=\mathrm{O})$, $190.00(\mathrm{C}=\mathrm{S}) \mathrm{ppm}$, Anal. Calcd. for $\mathrm{C}_{10} \mathrm{H}_{8} \mathrm{~N}_{2} \mathrm{O}_{2} \mathrm{~S}_{2}(\mathrm{M}=252)$ : C, 47.60, H, 3.17, N, 11.20. Found: C, 47.46, H, 3.38, N, 11.08.

\section{Methoxycarbonylmethyl 3-(4-chlorobenzylidene)-dithiocarbazate (3a)}

This compound was obtained as pale yellow crystalline, mp 139-141 ${ }^{\circ} \mathrm{C}$; IR $\widetilde{v}$ (KBr disc): CO 1712, CN(imine) $1582(\mathrm{~s}) \mathrm{cm}^{-1}$; ${ }^{1} \mathrm{H}$ NMR (d $6^{-}$DMSO): $\delta=3.65$ (s, $\left.3 \mathrm{H}, \mathrm{OCH}_{3}\right), 4.15$ $\left(\mathrm{s}, 2 \mathrm{H}, \mathrm{CH}_{2}\right), 7.55(\mathrm{~d}, 2 \mathrm{H}, \mathrm{Ar}, \mathrm{J}=8.16), 7.76(\mathrm{~d}, 2 \mathrm{H}, \mathrm{Ar}, \mathrm{J}=8.13), 8.25(\mathrm{~s}, 1 \mathrm{H}, \mathrm{CH}=\mathrm{N}) \mathrm{ppm}$.

${ }^{13} \mathrm{C}$ NMR (d 6 - DMSO): $\delta=36.25\left(1 \mathrm{C}, \mathrm{CH}_{2}\right), 53.17\left(1 \mathrm{C}, \mathrm{OCH}_{3}\right)$, (amine fragment), 130.01, 130.04, 133.03, 136.31, 138.67(6C, Ar), 146.51 (1C, $\mathrm{C}=\mathrm{N}$, imine), 169.76(C=O), 197.78 $(\mathrm{C}=\mathrm{S})$ ppm. Anal. Calcd. for $\mathrm{C}_{11} \mathrm{H}_{11} \mathrm{ClN}_{2} \mathrm{O}_{2} \mathrm{~S}_{2}(\mathrm{M}=302.7)$ : $\mathrm{C}, 43.60, \mathrm{H}, 3.63, \mathrm{~N}, 9.25$. Found: C, 43.18, H, 3.56, N, 9.36.

\section{Methoxycarbonylmethyl 3-(3-nitrobenzylidene)-dithiocarbazate (3b)}

Mp 139-141 ${ }^{\circ} \mathrm{C}$; IR $\widetilde{v}$ (KBr disc): CO 1719, CN(imine) $1602(\mathrm{~m}), \mathrm{NO}_{2}(1513,1340) \mathrm{cm}^{-1}$; ${ }^{1} \mathrm{H}$ NMR $\left(\mathrm{d}_{6}-\mathrm{DMSO}\right): \delta=\delta=3.66\left(\mathrm{~s}, 3 \mathrm{H}, \mathrm{OCH}_{3}\right), 4.16\left(\mathrm{~s}, 2 \mathrm{H}, \mathrm{CH}_{2}\right), 7.73-8.37(4 \mathrm{H}, \mathrm{Ar})$, $8.54(\mathrm{~s}, 1 \mathrm{H}, \mathrm{CH}=\mathrm{N}) \mathrm{ppm},{ }^{13} \mathrm{C} \mathrm{NMR}\left(\mathrm{d}_{6}-\mathrm{DMSO}\right): \delta=36.29\left(\mathrm{C}, \mathrm{CH}_{2}\right.$ (amine fragment), $53.18\left(1 \mathrm{C}, \mathrm{OCH}_{3)}, 122.35,125.87,131.53,134.49,135.97,145.44(6 \mathrm{C}, \mathrm{Ar}), 149.15\right.$ (C, C=N, imine), $169.70(\mathrm{C}=\mathrm{O}), 197.74(\mathrm{C}=\mathrm{S}) \mathrm{ppm}$. Anal. Calcd. for $\mathrm{C}_{11} \mathrm{H}_{11} \mathrm{~N}_{3} \mathrm{O}_{4} \mathrm{~S}_{2}$ $(\mathrm{M}=313): \mathrm{C}, 42.17, \mathrm{H}, 3.51, \mathrm{~N}, 13.42$. Found: $\mathrm{C}, 42.08, \mathrm{H}, 3.49$, N, 13.63.

\section{Methoxycarbonylmethyl 3-(4-methoxybenzylidene)-dithiocarbazate (3c)}

Mp 167-169 ${ }^{\circ} \mathrm{C}$; IR $\widetilde{v}$ (KBr disc): CO 1705, CN(imine) $1616(\mathrm{~s}) \mathrm{cm}^{-1} ;{ }^{1} \mathrm{H}$ NMR (d $6^{-}$DMSO): $\delta=3.86\left(\mathrm{~s}, 3 \mathrm{H}, \mathrm{OCH}_{3}\right), 4.35\left(\mathrm{~s}, 2 \mathrm{H}, \mathrm{CH}_{2}\right), 7.11(\mathrm{~d}, 1 \mathrm{H}, \mathrm{Ar}, \mathrm{J}=5.4), 7.86(\mathrm{~d}, 1 \mathrm{H}, \mathrm{Ar}, \mathrm{J}=5.1)$, $8.60(\mathrm{~s}, 1 \mathrm{H}, \mathrm{CH}=\mathrm{N}) \mathrm{ppm},{ }^{13} \mathrm{C} \mathrm{NMR}\left(\mathrm{d}_{6}-\mathrm{DMSO}\right): \delta=35.55\left(\mathrm{C}, \mathrm{CH}_{2}\right.$ (amine fragment), $56.45\left(\mathrm{C}, \mathrm{OCH}_{3}\right), 115.56,125.17,131.82,164.03(6 \mathrm{C}, \mathrm{Ar}), 170.63(\mathrm{C}, \mathrm{C}=\mathrm{N}$, imine $), 171.15$ $(\mathrm{C}=\mathrm{O}), 197.69(\mathrm{C}=\mathrm{S}) \mathrm{ppm}$. Anal. Calcd. for $\mathrm{C}_{12} \mathrm{H}_{14} \mathrm{~N}_{2} \mathrm{O}_{3} \mathrm{~S}_{2}(\mathrm{M}=298)$ : C, 48.32, $\mathrm{H}, 4.70, \mathrm{~N}$, 9.39. Found: C, 48.35, H, 4.57, N, 9.33. 
Methoxycarbonylmethyl 3-(2- hedroxy-3-methoxybenzylidene)-dithiocarbazate (3d)

Mp 173-174 ${ }^{\circ} \mathrm{C}$; IR $\widetilde{v}$ (KBr disc): CO 1706, CN(imine) 1616(s) $\mathrm{cm}^{-1} ;{ }^{1} \mathrm{H}$ NMR (d $\mathrm{d}^{-}$DMSO): $\delta=3.65\left(\mathrm{~s}, 3 \mathrm{H}, \mathrm{OCH}_{3}\right), \delta=3.82\left(\mathrm{~s}, 3 \mathrm{H}, \mathrm{OCH}_{3}\right), 4.15\left(\mathrm{~s}, 2 \mathrm{H}, \mathrm{CH}_{2}\right), 6.84(\mathrm{~m}, 1 \mathrm{H}, \mathrm{Ar}), 7.04(\mathrm{~d}$, $1 \mathrm{H}, \mathrm{Ar}, \mathrm{J}=7.8), 7.30(\mathrm{~d}, 1 \mathrm{H}, \mathrm{Ar}, \mathrm{J}=7.73), 8.58(1 \mathrm{H}, \mathrm{CH}=\mathrm{N}), 9.60(1 \mathrm{H}, \mathrm{OH}) \mathrm{ppm},{ }^{13} \mathrm{C} \mathrm{NMR}$ $\left(\mathrm{d}_{6}-\mathrm{DMSO}\right): \delta=35.82\left(\mathrm{C}, \mathrm{CH}_{2}\right.$ (amine fragment), $52.75\left(\mathrm{C}, \mathrm{OCH}_{3}\right), 56.36\left(\mathrm{C}, \mathrm{OCH}_{3}\right)$, 114.37, 118.35, 119.86, 119.98, 144.85, 147.45 (6C, Ar), $148.56(\mathrm{C}, \mathrm{C}=\mathrm{N}$, imine $), 169.40$ $(\mathrm{C}=\mathrm{O}), 195.44(\mathrm{C}=\mathrm{S}) \mathrm{ppm}$. Anal. Calcd. for $\mathrm{C}_{12} \mathrm{H}_{14} \mathrm{~N}_{2} \mathrm{O}_{4} \mathrm{~S}_{2}(\mathrm{M}=314): \mathrm{C}, 45.86, \mathrm{H}, 4.46, \mathrm{~N}$, 8.92. Found: $\mathrm{C}, 45.81, \mathrm{H}, 4.48, \mathrm{~N}, 8.81$.

\section{Methoxycarbonylmethyl 3-(4- hedroxybenzylidene)-dithiocarbazate (3e)}

Mp 185-186 ${ }^{\circ} \mathrm{C}$; IR $\tilde{\mathrm{v}}$ ( $\mathrm{KBr}$ disc): $\mathrm{CO} 1708, \mathrm{CN}\left(\right.$ imine) $1575(\mathrm{~s}) \mathrm{cm}^{-1} ;{ }^{1} \mathrm{H} \mathrm{NMR}\left(\mathrm{d}_{6}{ }^{-}\right.$ DMSO): $\delta=3.64\left(\mathrm{~s}, 3 \mathrm{H}, \mathrm{OCH}_{3}\right), 4.08\left(\mathrm{~s}, 2 \mathrm{H}, \mathrm{CH}_{2}, 6.43(\mathrm{~d}, 2 \mathrm{H}, \mathrm{Ar}, \mathrm{J}=8.0), 7.57\right.$ (d, 2H, Ar, $\mathrm{J}=8.0), 8.15(1 \mathrm{H}, \mathrm{CH}=\mathrm{N}), 10.13(1 \mathrm{H}, \mathrm{OH}) \mathrm{ppm},{ }^{13} \mathrm{CNMR}\left(\mathrm{d}_{6}-\mathrm{DMSO}\right):,{ }^{13} \mathrm{C}$ NMR $\left(\mathrm{d}_{6}-\right.$ DMSO): $\delta=35.76\left(\mathrm{C}, \mathrm{CH}_{2}\right.$ (amine fragment), $52.71\left(\mathrm{C}, \mathrm{OCH}_{3}\right), 116.71,124.50,129.96$, 160.77 (6C, Ar), $147.80(\mathrm{C}, \mathrm{C}=\mathrm{N}$, imine), $169.50(\mathrm{C}=\mathrm{O}), 194.96(\mathrm{C}=\mathrm{S}) \mathrm{ppm}$. Anal. Calcd. for $\mathrm{C}_{11} \mathrm{H}_{12} \mathrm{~N}_{2} \mathrm{O}_{3} \mathrm{~S}_{2}(\mathrm{M}=284)$ : C, 46.48, H, 4.22, N, 9.86. Found: C, 46.26, H, 4.28, N, 9.75.

\section{Results and Discussion}

Reaction of $\mathrm{N}$-aminorhodanine with a series of aromatic aldehydes was investigated to afford the corresponding imines (2a-3e and 3a-3e) (Scheme 1). The results are listed in Table 1.

Table 1. Synthesis of imines of $N$-aminorhodanine under different condition

\begin{tabular}{|c|c|c|c|c|c|c|c|c|c|}
\hline \multirow{2}{*}{ Aldehyde } & \multicolumn{3}{|c|}{$\begin{array}{l}\mathrm{CH}_{3} \mathrm{CN}(\mathrm{HCl}, \\
\left.\mathrm{CH}_{3} \mathrm{COOH}\right)\end{array}$} & \multicolumn{3}{|c|}{$\mathrm{CH}_{3} \mathrm{OH}(\mathrm{HCl})$} & \multicolumn{3}{|c|}{$\begin{array}{l}\mathrm{Al}_{2} \mathrm{O}_{3}, \mathrm{MV} \\
\text { irradiation }\end{array}$} \\
\hline & Entry & $\begin{array}{c}\text { Time, } \\
\mathrm{h}\end{array}$ & $\begin{array}{c}\text { Yield, } \\
\%\end{array}$ & Entry & $\begin{array}{c}\text { Time, } \\
\mathrm{h}\end{array}$ & $\begin{array}{c}\text { Yield, } \\
\%\end{array}$ & Entry & $\begin{array}{c}\text { Time } \\
\text { Sec }\end{array}$ & $\begin{array}{c}\text { Yield, } \\
\%\end{array}$ \\
\hline 4-Chlorobe & $2 \mathbf{a}$ & 8 & $>80$ & $\mathbf{3 a}$ & 8 & 82 & $2 \mathbf{2 a}$ & 220 & 84 \\
\hline 3-Nitrobenzaldehyde & $2 \mathbf{b}$ & 8 & $>80$ & 3b & 8 & 85 & $2 \mathbf{b}$ & 340 & 85 \\
\hline 4-Methoxybenzaldehyde & $2 c$ & 8 & $>80$ & $3 c$ & 8 & 82 & $2 c$ & 220 & 79 \\
\hline $\begin{array}{l}\text { 2-Hydroxy-3- } \\
\text { methoxybenzaldehyde }\end{array}$ & 2d & 8 & $>80$ & 3d & 8 & 78 & 2d & 240 & 76 \\
\hline 4-Hydroxybenzaldehyde & $2 \mathrm{e}$ & 8 & $>80$ & $3 e$ & 8 & 81 & $2 \mathrm{e}$ & 180 & 70 \\
\hline
\end{tabular}

As shown in Scheme 1, reactions of $\mathrm{N}$-aminorhodanine with aldehyde in $\mathrm{CH}_{3} \mathrm{CN}$, $\mathrm{MeOH}\left(\mathrm{CH}_{3} \mathrm{COOH}\right.$ as acidic catalyst) or on $\mathrm{Al}_{2} \mathrm{O}_{3}$ under microwave irradiation led to 2 (2a-2e), while reactions were performed in solvent such as methanol $\mathbf{3}$ (3a-3e) were formed. The IR spectra of all compounds show a characteristic band in the region of $1595-1610 \mathrm{~cm}^{-1}$, which can be assigned as stretching vibration bands for azomethine group $(v \mathrm{~N}=\mathrm{CH})$. The similar range is also reported in literature ${ }^{8,10} .{ }^{1} \mathrm{H}$ NMR spectra of the compounds showed a sharp signal (singlet) in the region of 8.01- $9.10 \mathrm{ppm}$, which can be assigned to the azomethine proton ${ }^{9,10}$. In addition to normal signals in ${ }^{1} \mathrm{H}$ NMR spectra of 2 and 3, $\mathrm{OCH}_{3}$ signals of 3a-3e are obtained in the region of 3.61-3.6 ppm and ${ }^{13} \mathrm{C} \mathrm{NMR}$ spectra showed a signal at in the region of 52.71-53.17 ppm which can be assigned to the $\mathrm{OCH}_{3}$ group. As shown in scheme 2, $\mathrm{HCl}$ plays an important role to protonation of carbonyl group and activation of carbon for nucleophilic attack of methanol oxygen, followed by ring opening of $\mathrm{N}$-aminorhodanine. Recently we reported the same ring opening reaction in the reaction of $\mathrm{N}$-aminorhodanine and 2-cholorobenzaldehyde in ethanol and in the presence of $\mathrm{HCl}^{11}$. 

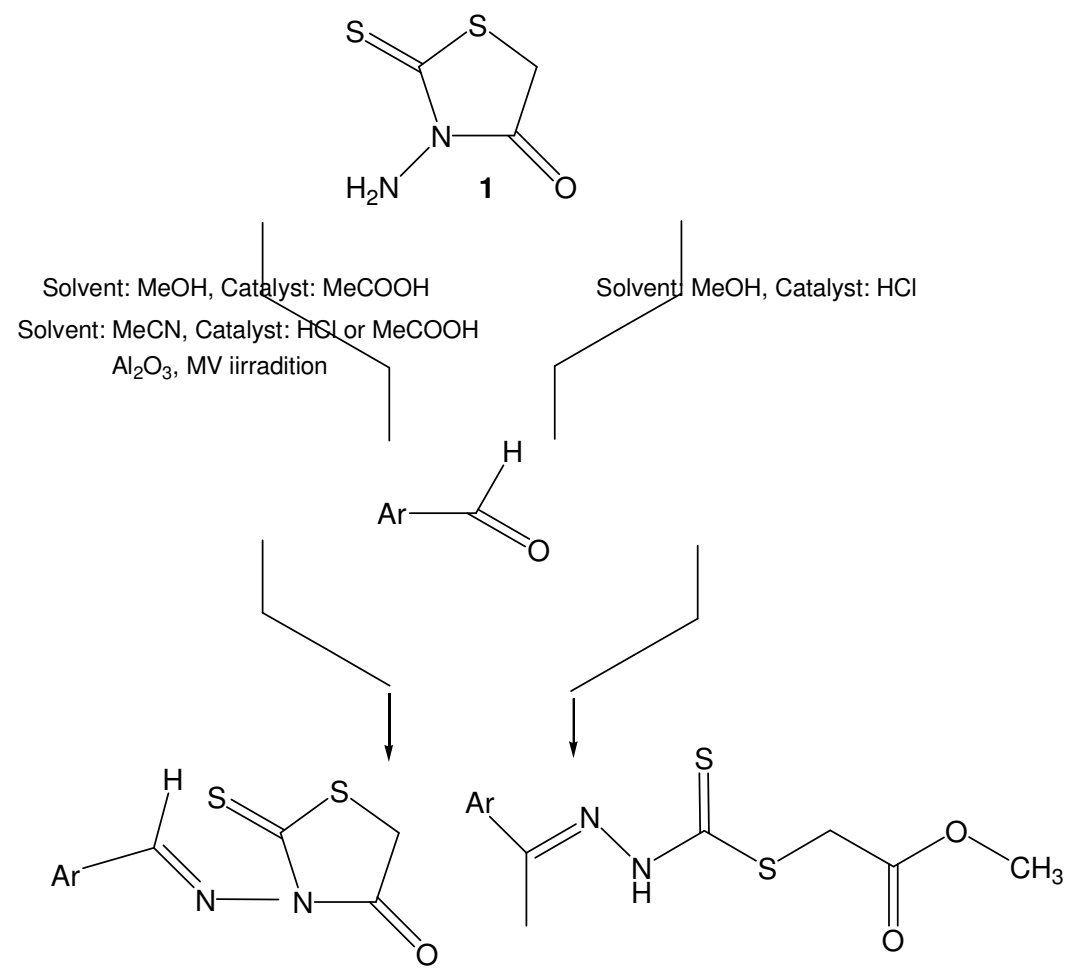

2

3

Scheme 1. Reaction of $N$-aminorhodanine with aromatic aldehydes under different conditions<smiles>COC(=O)C1CSC(=S)N1N</smiles>

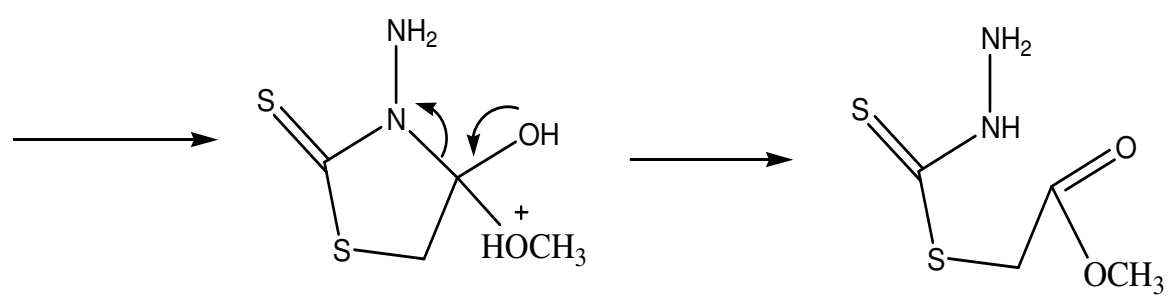

Scheme 2

\section{Acknowledgment}

The author is grateful to Scientific Society of Nanotechnology, Islamic Azad University, Yazd Branch, for the support of this work. 


\section{References}

1. Demirbas A, Turk J Chem., 2004, 28, 311-324.

2. Rando D G, Sato D N, Siqueira L, Malvezzi A, Leite C Q F, Amaral A T, Ferreira E I and Tavares L C, Bioorg Med Chem., 2002, 10, 557-560.

3. Demirb N and Demirbas A U, Gurluoglu R and Demirb A, Bioorg Med Chem., 2002, 10, 3717.

4. Todeschini A.R, Miranda A N, Silva K C M, Parrini S C and Barreiro E, Eur J Med Chem., 1998, 33, 189-199.

5. $\quad$ Reddy H and Lirgappa Y, Indian J Heterocycl Chem., 1994, 33, 919-923.

6. Galic N, Peric B, Kojic-Prodic B and Cimerman Z, J Mol Stuc., 2001, 559, 187-194.

7. Wyrzykiewicz E. and Prukah D, J Heterocycl Chem., 1998, 35, 381-387.

8. Tabatabaee M, Ghassemzadeh M, Zarabi B, Neumüller B, Z Naturforsch., 2006, 61b, $1421-1425$

9. Hayes B L, Aldrichimica Acta., 2004, 37, 66-77.

10. Tabatabaee M, Ghassemzadeh M, Zarabi B, Heravi M M, Anary-Abbasinejad M and Neumüller B, Phosphorus, Sulfur Silicon Relat Elem., 2007, 182, 677-686.

11. Tabatabaee M, Ghassemzadeh M, Dehghan A R, Khavasi H R and Heravi M M, Acta Cryst., 2007, E63, o42-o43. 


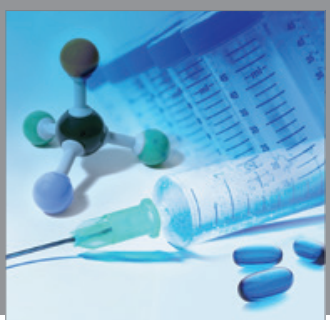

International Journal of

Medicinal Chemistry

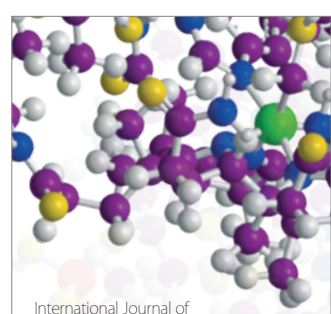

Carbohydrate Chemistry

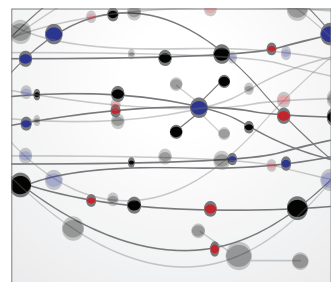

The Scientific World Journal
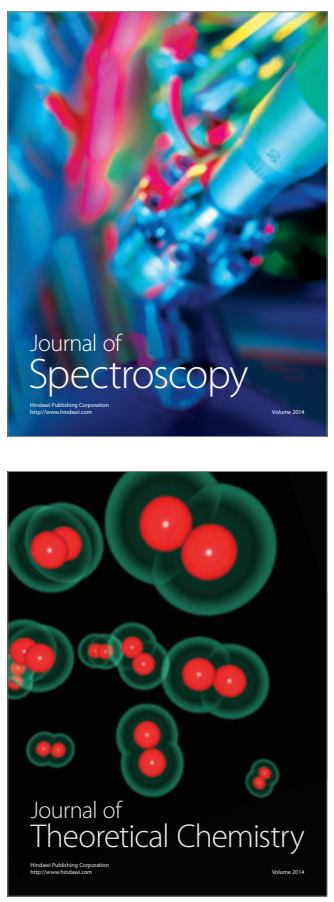
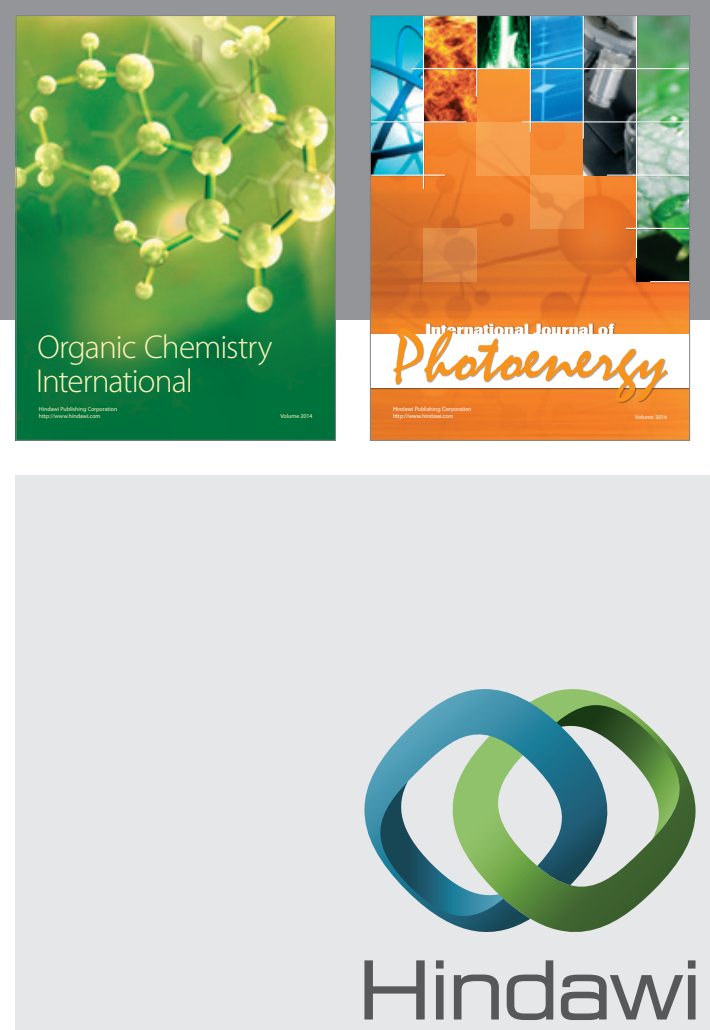

Submit your manuscripts at

http://www.hindawi.com
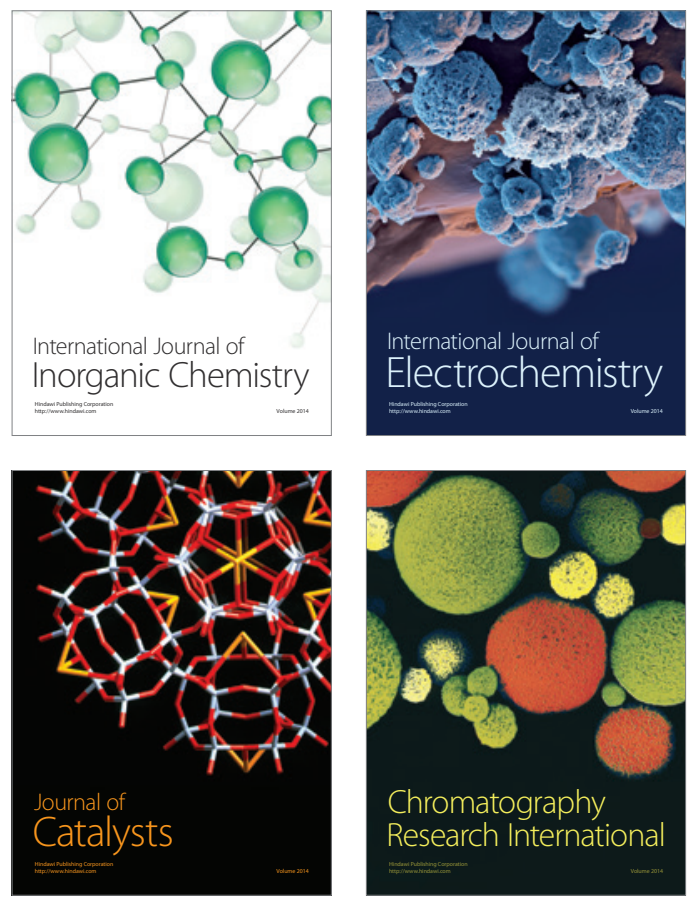
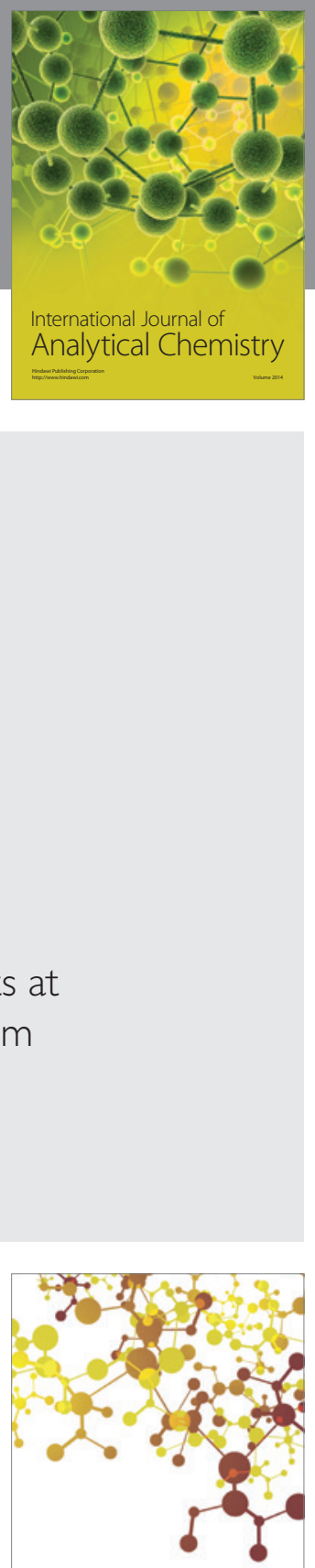

Journal of

Applied Chemistry
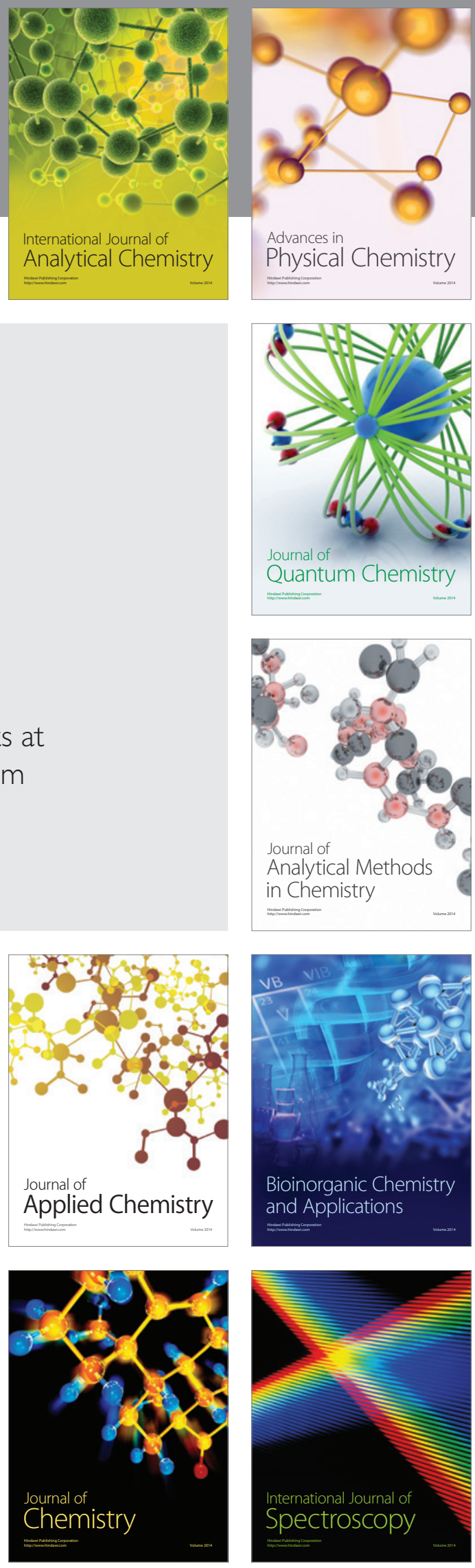